\title{
Hypothesis Support for Information Integration in Four-Valued Logics
}

\author{
Yann Loyer, Nicolas Spyratos ${ }^{\star}$, and Daniel Stamate \\ Laboratoire de Recherche en Informatique, UMR 8623, \\ Université de Paris Sud, Bat. 490, 91405 Orsay \\ \{loyer, spyratos, daniel\}@lri.fr
}

\begin{abstract}
We address the problem of integrating information coming from different sources. The information consists of facts that a central server collects and tries to combine using (a) a set of logical rules, i.e. a logic program, and (b) a hypothesis representing the server's own estimates. In such a setting incomplete information from a source or contradictory information from different sources necessitate the use of many-valued logics in which programs can be evaluated and hypotheses can be tested. To carry out such activities we propose a formal framework based on Belnap's four-valued logic. In this framework we work with the class of programs defined by Fitting and we develop a theory for information integration. We also establish an intuitively appealing connection between our hypothesis testing mechanism on the one hand, and the well-founded semantics and Kripke-Kleene semantics of Datalog programs with negation, on the other hand.
\end{abstract}

Keywords : deductive databases and knowledge bases, information integration, logics of knowledge, inconsistency, four-valued logic.

\section{Introduction}

In several information oriented activities there is a need for combining (or "integrating") information coming from different sources.

A typical example of such information-oriented activity is building a data warehouse, i.e. a special kind of very large database for decision-making support in big enterprises [1. The information stored in a data warehouse is obtained from queries to operational databases internal to the enterprise, and from remote information sources external to the enterprise accessed through the Internet. The answers to all such queries are then combined (by the so-called "integrator") to derive the information to be stored in the data warehouse.

The basic pattern of the data warehouse paradigm, i.e. collection of information then integration, is encountered in many different situations. What changes usually from one situation to another is the type (and volume) of the collected

\footnotetext{
* Part of this research work was conducted while this author was visiting with the Meme Media Laboratory, University of Hokkaido, Japan.
} 
information and the means used for the integration.

In this paper we address a specific problem of information integration, namely, the information consists of facts that a central server collects from a number of autonomous sources and then tries to combine them using:

- a set of logical rules, i.e. a logic program, and

- a hypothesis, representing the server's own estimates.

In such a setting incomplete information from a source or contradictory information coming from different sources necessitate the use of many-valued logics, in which programs can be evaluated and hypotheses can be tested. Let us see a simple example.

Example 1. Consider a legal case where a judge (the "central server") has to decide whether to charge a person named John accused of murder. To do so, the judge first collects facts from two different sources: the public prosecutor and the person's lawyer. The judge then combines the collected facts using a set of rules in order to reach a decision. For the sake of our example let us suppose that the judge has collected a set of facts $F$ that he combines using a set of rules $R$ as follows:

$$
\begin{aligned}
& F \quad\left[\begin{array}{cc}
\text { witness (John) } & \text { friends(John, Ted) } \\
\downarrow & \downarrow \\
\text { false } & \text { true }
\end{array}\right] \\
& R\left\{\begin{array}{l}
\operatorname{suspect}(\mathrm{X}) \leftarrow \operatorname{motive}(X) \vee \text { witness }(X) \\
\operatorname{innocent}(X) \leftarrow \exists Y(\operatorname{alibi}(X, Y) \wedge \neg \text { friends }(X, Y)) \\
\text { friends }(X, Y) \leftarrow \operatorname{friends}(Y, X) \vee(\text { friends }(X, Z) \wedge \text { friends }(Z, Y)) \\
\operatorname{charge}(X) \leftarrow \operatorname{suspect}(X) \oplus \neg \operatorname{innocent}(X)
\end{array}\right.
\end{aligned}
$$

The first fact of $F$ says that there is no witness, i.e. the fact witness(John) is false. The second fact of $F$ says that Ted is a friend of John, i.e. the fact friends $(J o h n, T e d)$ is true.

Turning now to the set of rules, the first rule of $R$ describes how the prosecutor works: in order to support the claim that a person $X$ is a suspect, the prosecutor tries to provide a motive and/or a witness.

The second rule of $R$ describes how the lawyer works: in order to support the claim that $X$ is innocent, the lawyer tries to provide an alibi for $X$ by a person who is not a friend of $X$. This rule depends on the third rule which defines the relation friends.

Finally, the fourth rule of $R$ is the "decision making rule" and describes how the judge works: in order to reach a decision as to whether to charge $X$, the judge examines the premises suspect $(X)$ and $\neg$ innocent $(X)$. As explained earlier, the values of these premises come from two different sources: the prosecutor and the lawyer. Each of these premises can have the value true or false. However, it is also possible that the value of a premiss is undefined. For example, if a motive is 
not known and a witness has not been found, then the value of $\operatorname{suspect}(\mathrm{X})$ will be undefined.

In view of these observations, the question is what value is appropriate to associate with charge $(\mathrm{X})$.

What we propose is to collect together the values of the premises $\operatorname{suspect}(\mathrm{X})$ and $\rightarrow$ innocent $(\mathrm{X})$, and to consider the resulting set of values as the value of charge $(\mathrm{X})$. This is precisely what the notation

$$
\operatorname{charge}(X) \leftarrow \operatorname{suspect}(X) \oplus \neg \operatorname{innocent}(X)
$$

means, where $\oplus$ denotes the "collecting together" operation.

It follows that there are four possible values for charge $(\mathrm{X}): \emptyset,\{$ true $\},\{$ false $\}$ and $\{$ true, false $\}$. We shall call these values : Underdefined, True, False and Overdefined, and we shall denote them by $\mathcal{U}, \mathcal{T}, \mathcal{F}$ and $\mathcal{O}$, respectively.

The value Underdefined for a premiss means that the premiss is true or false but its actual value is currently unknown. For the purpose of this paper we shall assume that any premiss whose value is not known is associated with the value Underdefined.

We note that the value Underdefined is related to the so-called "null values" of attributes in database theory. In database theory, however, a distinction is made between two types of null values [13]:

- the attribute value exists but is currently unknown

- the attribute value does not exist

An example of the first type is the Department-value for an employee that has just been hired but has not yet been assigned to a specific department, and an example of the second type is the maiden name of a male employee. The value Underdefined corresponds to the first type of null value.

Returning now to our example, the decision whether to charge John depends on the value that charge(John) will receive when collecting the values of the premises together. Looking at the facts of $F$ and the rules of $R$ (and using intuition) we can see that suspect(John) and innocent(John) both receive the value $\mathcal{U}$ and so then does charge(John).

This is clearly a case where the judge cannot decide whether to actually charge John!

In the context of decision making, however, one has to reach a decision (based on the available facts and rules) even if some values are not defined. This can be accomplished by assuming values for some or all underdefined premises. Such an assignment of values to underdefined premises is what we call a hypothesis.

Thus in our example, if the judge assumes the innocence of John, then charge(John) receives the value false and John is not charged. We note that this is precisely what happens in real life under similar circumstances, i.e. the defendant is assumed innocent until proved guilty.

Clearly, when hypothesizing on underdefined premises we would like our hypothesis to be "reasonable" in some sense, with respect to the available informa- 
tion, i.e., with respect to the given facts and rules. Roughly speaking, we define a hypothesis $H$ to be "reasonable" or sound using the following test : calling a fact $f$ defined under $H$ if $H(f) \neq \mathcal{U}$,

1. add $H$ to $F$ to produce a new set of facts $F^{\prime}=F \cup H$;

2. apply the rules of $R$ to $F^{\prime}$ to produce a new assignment of values $H^{\prime}$;

3 . if the facts defined under $H$ are assigned to the same values in $H^{\prime}$ then $H$ is sound, otherwise $H$ is not sound.

That is, if there is no fact of $H$ that has changed value as a result of rule application then $H$ is a sound hypothesis; otherwise $H$ is unsound.

In our example, for instance, consider the following hypothesis:

$$
H_{1}=\left[\begin{array}{cc}
\text { innocent(John) } & \text { charge (John) } \\
\downarrow & \downarrow \\
\mathcal{T} & \mathcal{T}
\end{array}\right]
$$

Applying the above test we find the following values for the facts of $H_{1}$ :

$$
H_{1}^{\prime}=\left[\begin{array}{cc}
\text { innocent(John) } & \text { charge (John) } \\
\downarrow & \downarrow \\
\mathcal{T} & \mathcal{F}
\end{array}\right]
$$

As we can see, the fact charge(John) has changed value, i.e. this fact had the value $\mathcal{T}$ in $H_{1}$ and now has the value $\mathcal{F}$ in $H_{1}^{\prime}$. Therefore, $H_{1}$ is not a sound hypothesis.

Next, consider the following hypothesis:

$$
H_{2}=\left[\begin{array}{cc}
\text { innocent }(J o h n) & \text { charge (John) } \\
\downarrow & \downarrow \\
\mathcal{T} & \mathcal{F}
\end{array}\right]
$$

Applying again our test we find :

$$
H_{2}^{\prime}=\left[\begin{array}{cc}
\text { innocent(John) } & \text { charge(John) } \\
\downarrow & \downarrow \\
\mathcal{T} & \mathcal{F}
\end{array}\right]
$$

That is, the values of the facts of $H_{2}$ remain unchanged in $H_{2}^{\prime}$, thus $H_{2}$ is a sound hypothesis.

Intuitively, if our hypothesis is sound this means that what we have assumed is compatible with the given facts and rules.

From now on let us denote $\mathcal{P}$ the facts of $F$ together with the rules of $R$, i.e. $\mathcal{P}=\langle F, R\rangle$, and let us call $\mathcal{P}$ a program.

In principle, we may assume or hypothesize values for every possible ground atom. However, given a program $\mathcal{P}$ and a hypothesis $H$, we cannot expect $H$ to be sound with respect to $\mathcal{P}$, in general. What we can expect is that some "part" of $H$ is sound with respect to $\mathcal{P}$.

More precisely, given two hypotheses $H$ and $H^{\prime}$, call $H$ a part of $H^{\prime}$, denoted $H \leq H^{\prime}$, if $H(f) \neq \mathcal{U}$ implies $H(f)=H^{\prime}(f)$, i.e., if $H$ agrees with $H^{\prime}$ on every 
defined fact. It is then natural to ask, given program $\mathcal{P}$ and hypothesis $H$, what is the maximal part of $H$ that is sound with respect to $\mathcal{P}$. We call this maximal part the support of $H$ by $\mathcal{P}$, and we denote it by $s_{\mathcal{P}}^{H}$. Intuitively, the support of $H$ indicates how much of $H$ can be assumed safely, i.e., remaining compatible with the facts and rules of $\mathcal{P}$.

We show that the support $s_{\mathcal{P}}^{H}$ can be used to define a hypothesis-based semantics of $\mathcal{P}=\langle F, R\rangle$, denoted by $\operatorname{sem}_{\mathcal{P}}^{H}$. This is done by a fixpoint computation that uses an immediate consequence operator $T$ as follows:

$-F_{0}=F$;

$-F_{i+1}=T\left(F_{i}\right) \oplus s_{\left\langle F_{i}, R\right\rangle}^{H}$.

We also show that there is an interesting connection between hypothesis based semantics and the semantics of Datalog programs with negation. More precisely, we show that if $\mathcal{P}$ is a Datalog program with negation then:

- if $H$ is the everywhere false hypothesis then $\operatorname{sem}_{\mathcal{P}}^{H}$ coincides with the wellfounded semantics of $\mathcal{P}$ [1112], and

- if $H$ is the everywhere underdefined hypothesis then $\operatorname{sem}_{\mathcal{P}}^{H}$ coincides with the Kripke-Kleene semantics of $\mathcal{P}$ [4].

As we shall see, these results allow us to extend the well-founded semantics and the Kripke-Kleene semantics of Datalog program with negation to the broader class of Fitting programs [6].

Motivation for this work comes from the area of knowledge acquisition, where contradictions may occur during the process of collecting knowledge from different experts. Indeed, in multi-agent systems, different agents may give different answers to the same query. It is then important to be able to process the answers so as to extract the maximum of information on which the various agents agree, or to detect the items on which the agents give conflicting answers.

Motivation also comes from the area of deductive databases. Updates leading to a certain degree of inconsistency should be allowed because inconsistency can lead to useful information, especially within the framework of distributed databases. In particular, Fuhr and Rölleke showed in 7] that hypermedia retrieval requires the handling of inconsistent information.

The remaining of the paper is organized as follows. In Section 2 we recall very briefly some definitions and notations from well-founded semantics, Belnap's logic $\mathcal{F O U} \mathcal{R}$ and Fitting programs. We then proceed, in Section 3, to define sound hypotheses and their support by a Fitting program $\mathcal{P}$; we also discuss computational issues and we present algorithms for computing the support of a hypothesis by a program $\mathcal{P}$ and the hypothesis-based semantics of $\mathcal{P}$. In Section 4 we show that the notion of support actually unifies the notions of well-founded semantics and Kripke-Kleene semantics and extends them from Datalog program with negation to the broader class of Fitting programs. Section 5 contains concluding remarks and suggestions for further research. Proofs of theorems are omitted due to lack of space. 


\section{Preliminaries}

\subsection{Three-Valued logics}

Well founded semantics Well-founded semantics of logic programs were first proposed in [11. In the approach of [11 an interpretation $I$ is a set of ground literals that does not contain literals of the form $A$ and $\neg A$. Now, if we consider an instantiated program $P$ defined as in [11], its well-founded semantics is defined using the following two operators on partial interpretations $I$ :

- the immediate consequence operator $T_{P}$, defined by

$$
T_{P}(I)=\{\operatorname{head}(r) \mid r \in P \wedge \forall B \in \operatorname{bod} y(r), B \in I\} \text {, and }
$$

- the unfounded operator $U_{P}$, where $U_{P}(I)$ is defined to be the greatest unfounded set with respect to the partial interpretation $I$.

We recall that a set of instantiated atoms $U$ is said to be unfounded with respect to $I$ if for all instantiated atoms $A \in U$ and for all rules $r \in P$ the following holds:

$$
\operatorname{head}(r)=A \Rightarrow \exists B \in \operatorname{body}(r)(\neg B \in I \vee B \in U)
$$

In 3. it is proven that $U_{P}(I)=\mathcal{H B} \backslash S P F_{P}(I)$, where $\mathcal{H B}$ is the Herbrand Base and $S P F_{P}(I)$ is the limit of the increasing sequence $\left[S P F^{i}(I)\right]_{i \geq 1}$ defined by:

$$
\begin{aligned}
-\operatorname{SPF}_{P}^{1}(I)=\{\operatorname{head}(r) \mid r \in P & \wedge \operatorname{pos}(\operatorname{body}(r))=\emptyset \\
& \wedge \forall B \in \operatorname{bod}(r), \neg B \notin I\} \\
-\operatorname{SPF}_{P}^{i+1}(I)=\{\operatorname{head}(r) \mid r \in P & \wedge \operatorname{pos}(\operatorname{body}(r)) \subseteq S P F_{P}^{i}(I) \\
& \wedge \forall B \in \operatorname{body}(r), \neg B \notin I\}, i>0 .
\end{aligned}
$$

The atoms of $S P F_{P}(I)$ are called potentially founded atoms.

The operator $W_{P}$, called the well-founded operator, is then defined by $W_{P}(I)$ $=T_{P}(I) \cup \neg U_{P}(I)$ and is shown to be monotone with respect to set inclusion. The well-founded semantics of $P$ is defined to be the least fixpoint of $W_{P}[11]$.

Kripke-Kleene semantics The Kripke-Kleene semantics was introduced in 44. In the approach of [4, a valuation is a function from the Herbrand base to the set of logical values $\{$ true, false, unknown\}. Now, given an instantiated program $\mathcal{P}$ defined as in [4, its Kripke-Kleene semantics is defined using an operator $\Phi$ on valuations, defined as follows : given a ground atom $A$,

- if there is a rule in $\mathcal{P}$ with head $A$, and the truth value of the body under $v$ is true, then $\Phi_{\mathcal{P}}(v)(A)=$ true;

- if there is a rule in $\mathcal{P}$ with head $A$, and for every rule in $\mathcal{P}$ with head $A$ the truth value of the body under $v$ is false, then $\Phi_{\mathcal{P}}(v)(A)=$ false;

- else $\Phi_{\mathcal{P}}(v)(A)=$ unknown. 


\section{$2.2 \quad$ Four-Valued Logics}

Belnap's four-valued logic In [2], Belnap defines a logic called $\mathcal{F O U} \mathcal{R}$ intended to deal with incomplete and inconsistent information. Belnap's logic uses four logical values that we shall denote by $\mathcal{F}, \mathcal{T}, \mathcal{U}$ and $\mathcal{O}$, i.e. $\mathcal{F} \mathcal{O U R}=\{\mathcal{F}$, $\mathcal{T}, \mathcal{U}, \mathcal{O}\}$. These values can be compared using two orderings, the knowledge ordering and the truth ordering.

In the knowledge ordering, denoted by $\leq_{k}$, the four values are ordered as follows: $\mathcal{U} \leq_{k} \mathcal{F}, \mathcal{U} \leq_{k} \mathcal{T}, \mathcal{F} \leq_{k} \mathcal{O}, \mathcal{T} \leq_{k} \mathcal{O}$. Intuitively, according to this ordering, each value of $\mathcal{F} \mathcal{O U} \mathcal{R}$ is seen as a possible knowledge that one can have about the truth of a given statement. More precisely, this knowledge is expressed as a set of classical truth values that hold for that statement. Thus, $\mathcal{F}$ is seen as $\{$ false $\}, \mathcal{T}$ is seen as $\{$ true $\}, \mathcal{U}$ is seen as $\emptyset$ and $\mathcal{O}$ is seen as $\{$ false,true $\}$. Following this viewpoint, the knowledge ordering is just the set inclusion ordering.

In the truth ordering, denoted by $\leq_{t}$, the four logical values are ordered as follows: $\mathcal{F} \leq_{t} \mathcal{U}, \mathcal{F} \leq_{t} \mathcal{O}, \mathcal{U} \leq_{t} \mathcal{T}, \mathcal{O} \leq_{t} \mathcal{T}$. Intuitively, according to this ordering, each value of $\mathcal{F} \mathcal{O U} \mathcal{R}$ is seen as the degree of truth of a given statement. $\mathcal{U}$ and $\mathcal{O}$ are both less false than $\mathcal{F}$, and less true than $\mathcal{T}$, but $\mathcal{U}$ and $\mathcal{O}$ are not comparable.

The two orderings are represented in the double Hasse diagram of Figure 1.

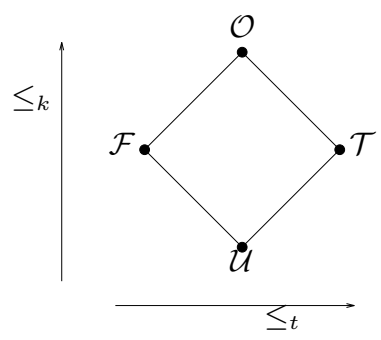

Fig. 1. The logic FOUR

Both $\leq_{t}$ and $\leq_{k}$ give $\mathcal{F} \mathcal{O U} \mathcal{R}$ a lattice structure. Meet and join under the truth ordering are denoted by $\wedge$ and $\vee$, and they are natural generalizations of the usual notions of conjunction and disjunction. In particular, $\mathcal{U} \wedge \mathcal{O}=\mathcal{F}$ and $\mathcal{U} \vee \mathcal{O}=\mathcal{T}$. Under the knowledge ordering, meet and join are denoted by $\otimes$ and $\oplus$, and are called the consensus and gullibility, respectively: $x \otimes y$ represents the maximal information on which $x$ and $y$ agree, whereas $x \oplus y$ adds the knowledge represented by $x$ to that represented by $y$. In particular, $\mathcal{F} \otimes \mathcal{T}=\mathcal{U}$ and $\mathcal{F} \oplus \mathcal{T}=$ $\mathcal{O}$.

There is a natural notion of negation in the truth ordering denoted by $\neg$, and we have: $\neg \mathcal{T}=\mathcal{F}, \neg \mathcal{F}=\mathcal{T}, \neg \mathcal{U}=\mathcal{U}, \neg \mathcal{O}=\mathcal{O}$. There is a similar notion for the knowledge ordering, called conflation, denoted by -, and we have: $-\mathcal{U}=$ $\mathcal{O},-\mathcal{O}=\mathcal{U},-\mathcal{F}=\mathcal{F},-\mathcal{T}=\mathcal{T}$. 
The operations $\vee, \wedge, \neg$ restricted to the values $\mathcal{T}$ and $\mathcal{F}$ are those of classical logic, and if we add to these operations and values the value $\mathcal{U}$ then they are those of Kleene's strong three-valued logic.

Fitting programs Conventional logic programming has the set $\{\mathcal{F}, \mathcal{T}\}$ as its intended space of truth values but since not every query may produce an answer partial models are often allowed (i.e. $\mathcal{U}$ is added). If we want to deal with inconsistency as well then $\mathcal{O}$ must be added. Thus Fitting asserts that $\mathcal{F O U} \mathcal{R}$ can be thought as the "home" of ordinary logic programming and extends the notion of logic program, as follows:

\section{Definition 1. (Fitting program)}

- A formula is an expression built up from literals and elements of $\mathcal{F O U R}$, using $\wedge, \vee, \otimes, \oplus, \exists, \forall$.

- A clause is of the form $P\left(x_{1}, \ldots, x_{n}\right) \longleftarrow \phi\left(x_{1}, \ldots, x_{n}\right)$, where the atomic formula $P\left(x_{1}, \ldots, x_{n}\right)$ is the head, and the formula $\phi\left(x_{1}, \ldots, x_{n}\right)$ is the body. It is assumed that the free variables of the body are among $x_{1}, \ldots, x_{n}$.

- A program is a finite set of clauses with no predicate letter appearing in the head of more than one clause (this apparent restriction causes no loss of generality [5]).

We shall represent a Fitting program as a pair $\langle F, R\rangle$ where $F$ is a function from the Herbrand base into $\mathcal{F O U R}$ and $R$ a set of clauses. This is possible because every fact can be seen as a rule of the form $A \leftarrow v$, where $A$ is an atom and $v$ is a value in $\mathcal{F O U R}$.

A Datalog program with negation can be seen as a Fitting program whose underlying truth-value space is the subset $\{\mathcal{F}, \mathcal{T}, \mathcal{U}\}$ of $\mathcal{F O U R}$ and which does not involve $\otimes, \oplus, \forall, \mathcal{U}, \mathcal{O}, \mathcal{F}$.

\section{Hypothesis Testing}

In the remaining of this paper, in order to simplify the presentation, we assume that all Fitting programs are instantiated programs. Moreover, we use the term "program" to mean "Fitting program", unless explicitly stated otherwise.

\subsection{Interpretations}

First, we introduce some terminology and notation that we shall use throughout the paper. Given a program $\mathcal{P}$, call interpretation of $\mathcal{P}$ any function $I$ over the Herbrand base $\mathcal{H B}_{\mathcal{P}}$ such that, for every atom $A$ of $\mathcal{H B}_{\mathcal{P}}, I(A)$ is a value from $\mathcal{F O U R}$.

Two interpretations $I$ and $J$ are compatible if, for every ground atom A, $(I(A) \neq \mathcal{U}$ and $J(A) \neq \mathcal{U}) \Rightarrow I(A)=J(A)$. 
An interpretation $I$ is a part of an interpretation $J$, denoted $I \leq J$, if $I(A) \neq$ $\mathcal{U}$ implies $I(A)=J(A)$, for every ground atom $A$. Clearly, the part-of relation just defined is a partial ordering on the set $\mathcal{V}(\mathcal{F O U} \mathcal{R})$ of all interpretations over $\mathcal{F O U} \mathcal{R}$. Given an interpretation $I$, we denote by $\operatorname{def}(I)$ the set of all ground atoms $A$ such that $I(A) \neq \mathcal{U}$. Moreover, if $S$ is any set of ground atoms, we define the restriction of $I$ to $S$, denoted by $I_{/ S}$ as follows: for all $A \in \mathcal{H B}_{\mathcal{P}}$,

$$
I_{/ S}(A)=\left\{\begin{array}{l}
I(A) \text { if } A \in S, \\
\mathcal{U}, \text { otherwise. }
\end{array}\right.
$$

The operations of $\mathcal{F} \mathcal{O U R}$ can be extended naturaly to $\mathcal{V}(\mathcal{F} \mathcal{O U} \mathcal{R})$ in the following way: $I \oplus J(A)=I(A) \oplus J(A)$ and similarly for the other operations.

The actions of interpretations can be extended from atoms to formulas as follows:

$-I(X \wedge Y)=I(X) \wedge I(Y)$, and similarly for the other operators,

- I ( $\exists x) \phi(x))=\bigvee_{t=\text { closedterm }} I(\phi(t))$, and

- I ( $\forall x) \phi(x))=\bigwedge_{t=\text { closedterm }}^{\text {closedterm }} I(\phi(t))$.

If $B$ is a closed formula then we say that $B$ evaluates to the logical value $\alpha$, with respect to an interpretation $I$, denoted by $B \equiv \alpha$ w.r.t. $I$ or by $B \equiv_{I} \alpha$, if $J(B)=\alpha$ for any interpretation $J$ such that $I \leq J$ (i.e. if the value of $B$ is equal to $\alpha$ with respect to the defined atoms of $I$ whatever the values of underdefined atoms could be). There are formulas $B$ in which underdefined atoms do not matter for the logical value that can be associated with $B$. For example let us take $B=A \vee C$ and let the interpretation $I$ be defined by $I(A)=\mathcal{U}, I(C)=\mathcal{T}$; then no matter how $A$ is interpreted $B$ is evaluated to $\mathcal{T}$, that is, $B \equiv_{I} \mathcal{T}$.

Given an interpretation $I$ let $I_{\mathcal{O}}$ be the interpretation defined by : if $I(A) \neq \mathcal{U}$ then $I_{\mathcal{O}}(A)=I(A)$ else $I_{\mathcal{O}}(A)=\mathcal{O}$, for every atom $A$. The following lemma provides a method of testing whether $B \equiv_{I} \alpha$, based on the interpretation $I_{\mathcal{O}}$.

Lemma 1. Given a closed formula $B, B \equiv_{I} \alpha$ iff $I(B)=\alpha$ and $I_{\mathcal{O}}(B)=\alpha$.

\subsection{The Support of a Hypothesis}

Given a program $P=\langle F, R\rangle$ we consider two ways of inferring information from $\mathcal{P}$. First by activating the rules of $R$ in order to derive new facts from those of $F$, through an immediate consequence operator $T$. Second, by a kind of default reasoning based on a given hypothesis.

The immediate consequence operator $T$ that we use takes as input the facts of $F$ and returns an interpretation $T(F)$, defined as follows: for all ground atoms $A$,

$$
T_{R}(F)(A)=\left\{\begin{array}{l}
\alpha \text { if } A \leftarrow B \in R \text { and } B \equiv_{F} \alpha \\
\mathcal{U}, \text { otherwise }
\end{array}\right.
$$

What we call a hypothesis is actually just an interpretation $H$. However, we use the term "hypothesis" to stress the fact that the values assigned by $H$ to the atoms of the Herbrand base are assumed values - and not values that have 
been computed using the facts and rules of the program. As such, a hypothesis $H$ must be tested against the "sure" knowledge provided by $\mathcal{P}$. The test consists of "adding" $H$ to $F$, then activating the rules of $\mathcal{P}$ (using $T$ ) to derive an interpretation $H^{\prime}$. If $H \leq H^{\prime}$, then the hypothesis $H$ is a sound one, i.e. the values defined by $H$ are not in contradiction with those defined by $\mathcal{P}$. Hence the following definition:

Definition 2 (Sound Hypothesis). Let $\mathcal{P}=\langle F, R\rangle$ be a program and $H$ a hypothesis. $H$ is sound w.r.t. $\mathcal{P}$ if

- F and $H$ are compatible, and

- $H_{/ \operatorname{Head}(\mathcal{P})} \leq T(F \oplus H)$, where $\operatorname{Head}(\mathcal{P})=\{\mathcal{A} \mid \exists \mathcal{A} \leftarrow \mathcal{B} \in \mathcal{P}\}$.

We use the restriction of $H$ to $\operatorname{Head}(\mathcal{P})$ before making the comparison with $T(F \oplus H)$ because all atoms which are not head of any rule of $\mathcal{P}$ will be assigned to the value $U$ nderdefined by $T(F \oplus H)$. Then $H$ and $T(F \oplus H)$ are compatible on these atoms.

Even if a hypothesis $H$ is not sound w.r.t. $\mathcal{P}$, it may be that some part of $H$ is sound w.r.t. $\mathcal{P}$. Of course, we are interested to know what is the maximal part of $H$ that is sound w.r.t. $\mathcal{P}$. We shall call this maximal part the "support" of $H$. To see that the maximal part of $H$ is unique (and thus that the support is a well-defined concept), we give the following lemma:

Lemma 2. If $H_{1}$ and $H_{2}$ are two sound parts of $H$ w.r.t. $\mathcal{P}$, then $H_{1} \oplus H_{2}$ is sound w.r.t. $\mathcal{P}$.

Thus the maximal sound part of $H$ is defined by $\bigoplus\left\{H^{\prime} \mid H^{\prime} \leq H\right.$ and $H^{\prime}$ is sound w.r.t. $\mathcal{P}$ \}.

Definition 3 (Support). Let $\mathcal{P}$ be a program and $H$ a hypothesis. The support of $H$ w.r.t. $\mathcal{P}$, denoted $s_{\mathcal{P}}^{H}$, is the maximal sound part of $H$ w.r.t. $\mathcal{P}$ (where maximality is understood w.r.t. the part-of ordering $\leq$ ).

We now give an algorithm for computing the support $s_{\mathcal{P}}^{H}$ of a hypothesis $H$ w.r.t. a program $\mathcal{P}$.

Consider the following sequence $\left\langle P F_{i}\right\rangle, i \geq 0$ :

$-P F_{0}=\emptyset$

$-P F_{i+1}=\left\{A \mid A \leftarrow B \in \mathcal{P}\right.$ and $B \not \equiv H(A)$ w.r.t. $\left.F \oplus H_{/\left(\mathcal{H} \mathcal{B}_{\mathcal{P}} \backslash P F_{i}\right) \backslash \operatorname{def}(F)}\right\}$ for all $i \geq 0$,

The intuition here is that we want to evaluate step by step the atoms that could potentially have a logical value different than their values in $H$. We have the following results:

Proposition 1. The sequence $\left\langle P F_{i}\right\rangle, i \geq 0$ is increasing with respect to set inclusion and it has a limit reached in a finite number of steps. This limit is denoted PF.

Theorem 1. Let $\mathcal{P}$ and $H$ be fixed. Then $s_{\mathcal{P}}^{H}=H_{/\left(\mathcal{H} \mathcal{B}_{\mathcal{P}} \backslash P F\right)}$. 


\section{Hypothesis Based Semantics}

As we explained earlier, given a program $P=\langle F, R\rangle$, we derive information in two ways: by activating the rules (i.e. by applying the immediate consequence operator $\mathrm{T}$ ) and by making a hypothesis $H$ and computing its support $s_{\mathcal{P}}^{H}$ w.r.t. $\mathcal{P}$. In the whole, the information that we derive comes from $T(F) \oplus s_{\mathcal{P}}^{H}$.

Proposition 2. The sequence $\left\langle F_{n}\right\rangle, n \geq 0$ defined by $F_{0}=F$ and $F_{n+1}=$ $T_{R}\left(F_{n}\right) \oplus s_{\left\langle F_{n}, R\right\rangle}^{H}$ is increasing with respect to $\leq$, so it has a limit denoted by $\operatorname{sem}_{\mathcal{P}}^{H}$.

We recall that an interpretation $I$ is a model of a program $\mathcal{P}$ if for every rule $A \leftarrow B$ of $\mathcal{P}, I(B) \leq_{t} I(A)$.

Proposition 3. The interpretation $\operatorname{sem}_{\mathcal{P}}^{H}$ is a model of $\mathcal{P}$.

This justifies the following definition of semantics for $\mathcal{P}$.

Definition 4 (H-semantics of $\mathcal{P})$. The interpretation $\operatorname{sem}_{\mathcal{P}}^{H}$ is defined to be the semantics of $\mathcal{P}$ w.r.t. $H$ or the $H$-semantics of $\mathcal{P}$.

Following this definition, any given program $\mathcal{P}$ can be associated with different semantics, one for each possible hypothesis $H$. Theorem 2 below asserts that this approach extends the usual semantics of Datalog programs with negation to a broader class of programs, namely the Fitting programs.

Two remarks are in order here before stating Theorem 2. First, if we restrict our attention to three values only, i.e. $\mathcal{F}, \mathcal{T}$ and $\mathcal{U}$, then our definition of interpretation is equivalent to the one used by Van Gelder et als [11, in the following sense: given an interpretation $I$ following our definition, the set $\{A \mid I(A)=\mathcal{T}\} \cup\{\neg A \mid I(A)=\mathcal{F}\}$ is a partial interpretation following [1]; conversely, given a partial interpretation $J$ following [1], the function $I$ defined by: $I(A)=\mathcal{T}$ if $A \in J, I(A)=\mathcal{F}$ if $\neg A \in J$, and $I(A)=\mathcal{U}$ otherwise, is an interpretation in our sense.

Second, if we restrict our attention to Datalog programs with negation (recall that the class of Fitting programs strictly contains the Datalog programs with negation) then the concept of sound interpretation for the everywhere false hypothesis reduces to that of unfounded set of Van Gelder et als [11. The difference is that the definition in [1] has rather a syntactic flavor, while ours has a semantic flavor. Moreover, our definition not only extends the concept of Unfounded set to four-valued logic, but also generalizes its definition to any given hypothesis $H$ (not just the everywhere false hypothesis).

Theorem 2. Let $\mathcal{P}$ be a Datalog programs with negation.

1. If $H_{\mathcal{F}}$ is the everywhere false hypothesis, then $\operatorname{sem}_{\mathcal{P}}^{H_{\mathcal{F}}}$ coincides with the well-founded semantics of $\mathcal{P}$;

2. If $H_{\mathcal{U}}$ is the everywhere underdefined hypothesis, then $\operatorname{sem}_{\mathcal{P}}^{H_{\mathcal{U}}}$ coincides with the Kripke-Kleene semantics of $\mathcal{P}$. 


\section{Concluding Remarks}

We have defined a formal framework for information integration based on hypothesis testing. A basic concept of this framework is the support provided by a program $\mathcal{P}=\langle F, R\rangle$ to a hypothesis $H$. The support of $H$ is the maximal part of $H$ that does not contradict the facts of $F$ or the facts derived from $F$ using the rules of $R$.

We have then used the concept of support to define hypothesis-based semantics for the class of Fitting programs, and we have given an algorithm for computing these semantics.

Finally, we have shown that our semantics extends the well-founded semantics and the Kripke-Kleene semantics to Belnap's four-valued logic, and also generalizes them in the following sense: if we restrict our attention to three-valued logics then for $H_{\mathcal{F}}$ the everywhere false interpretation our semantics reduces to the well-founded semantics, and for $H_{\mathcal{U}}$ the everywhere underdefined interpretation our semantics reduces to the Kripke-Kleene semantics.

We believe that hypothesis-based semantics can be useful not only in the context of information integration but also in the context of explanation-based systems. Indeed, assume that a given hypothesis $H$ turns out to be a part of the $H$-semantics of a program $\mathcal{P}$. Then $\mathcal{P}$ can be seen as an "explanation" of the hypothesis $H$. We are currently investigating several aspects of this explanation oriented viewpoint.

\section{References}

1. Data Warehousing, Flexible Views for Ever-changing Corporate Data, Communications of the ACM, volume 41, number 9, septembre 1998.

2. Belnap, N. D., Jr, A Useful Four-Valued Logic, in: J. M. Dunn and G. Epstein (eds.), Modern Uses of Multiple-valued Logic, D. Reichel, Dordrecht, 1977.

3. Bidoit N., Froideveaux C., Negation by default and unstratifiable logic programs, TCS, 78, (1991)

4. Fitting, M. C., A Kripke/Kleene Semantics for Logic Programs, J. Logic Programming, 2:295-312 (1985).

5. Fitting, M. C., Bilattices and the Semantics of Logic Programming, J. Logic Programming, 11:91-116 (1991).

6. Fitting, M. C., The Family of Stable Models, J. Logic Programming, 17:197-225 (1993).

7. Fuhr, N. and Rölleke, T., HySpirit - a Probabilistic Inference Engine for Hypermedia Retrieval in Large Databases, (1997).

8. Ginsberg, M. L., Multivalued Logics: a Uniform Approach to Reasoning in Artificial Intelligence, Computational Intelligence, 4:265-316, 1988.

9. Ginsberg, M. L., Bilattices and modal operators, J. of Logic Computation, 1:41-69, 1990.

10. Loyer, Y., Spyratos, N., Stamate, D., Computing and Comparing Semantics of Programs in Four-valued Logics, in: Proceedings of the 24th Symposium on Mathematical Foundations of Computer Science (MFCS'99), LNCS 1672, Springer Verlag, 1999. 
$548 \quad$ Y. Loyer, N. Spyratos, and D. Stamate

11. Van Gelder, A., Ross, K. A., Schlipf, J. S., Unfounded Set and Well-Founded Semantics for General Logic Programs, in: Proceedings of the Seventh Symposium on Principles of Database Systems, 221-230, 1988.

12. Van Gelder, A., Ross, K. A., Schlipf, J. S., The Well-Founded Semantics for General Logic Programs, J. ACM, 38:620-650, 1991.

13. Zaniolo, C., Database Relations with Null Values, Journal of Computer and System Sciences, 28: 142-166, 1984. 